\title{
SPACE FLIGHT MANIPULATOR TECHNOLOGIES AND REQUIREMENTS FOR THE NASA FLIGHT TELEROBOTIC SERVICER (FTS)
}

\author{
John T. Chladek \\ NASA - Johnson Space Center \\ Houston, TX 77058 \\ William M. Craver \\ Lockheed Engineering and Sciences Co. \\ 2400 Nasa Road 1, Houston, TX 77058
}

\begin{abstract}
NASA Headquarters' Office of Advanced Concepts and Technology (OACT) joined efforts with Johnson Space Center's (JSC) Automation and Robotics Division and Langley Research Center's (LaRC) Information Systems Division to capture the technologies developed during the canceled NASA Flight Telerobotic Servicer (FTS) program planned for use on Space Station Freedom. The recent FTS Technology Capture effort completed the build and testing of one flight qualifiable FTS manipulator, deliverable to JSC's Automation \& Robotics Division for environmental testing. The many robotic technologies developed to meet the 30 year space environment design requirements are discussed in this paper. The manipulator properties were to allow positioning control to one thousandths of an inch, with zero actuator backlash over a temperature range of -50 to +95 degrees $C$, and were to include impedance control and inertial de-coupling. Safety
\end{abstract}

and reliability requirements are discussed that were developed to allow a thirty year life in space with minimum maintenance. The system had to meet the safety requirements for hazardous payloads for operation in the Shuttle Payload Bay during demonstration test flights prior to Station use. A brief description is contained on an Orbiter based robotic experiment and operational application using the dexterous FTS manipulator operating on the end of the Shuttle Remote Manipulator Systems (SRMS) from ground control.

\section{Anticipated_Misslon Tasks}

The original FTS concept for Space Station Freedom (SSF) was to provide telerobotic assistance to enhance crew activity and safety, and to reduce crew EVA (Extra Vehicular Activity) activity. The first flight of the FTS manipulator systems would demonstrate several candidate tasks and would verify manipulator performance parameters. These first flight tasks included

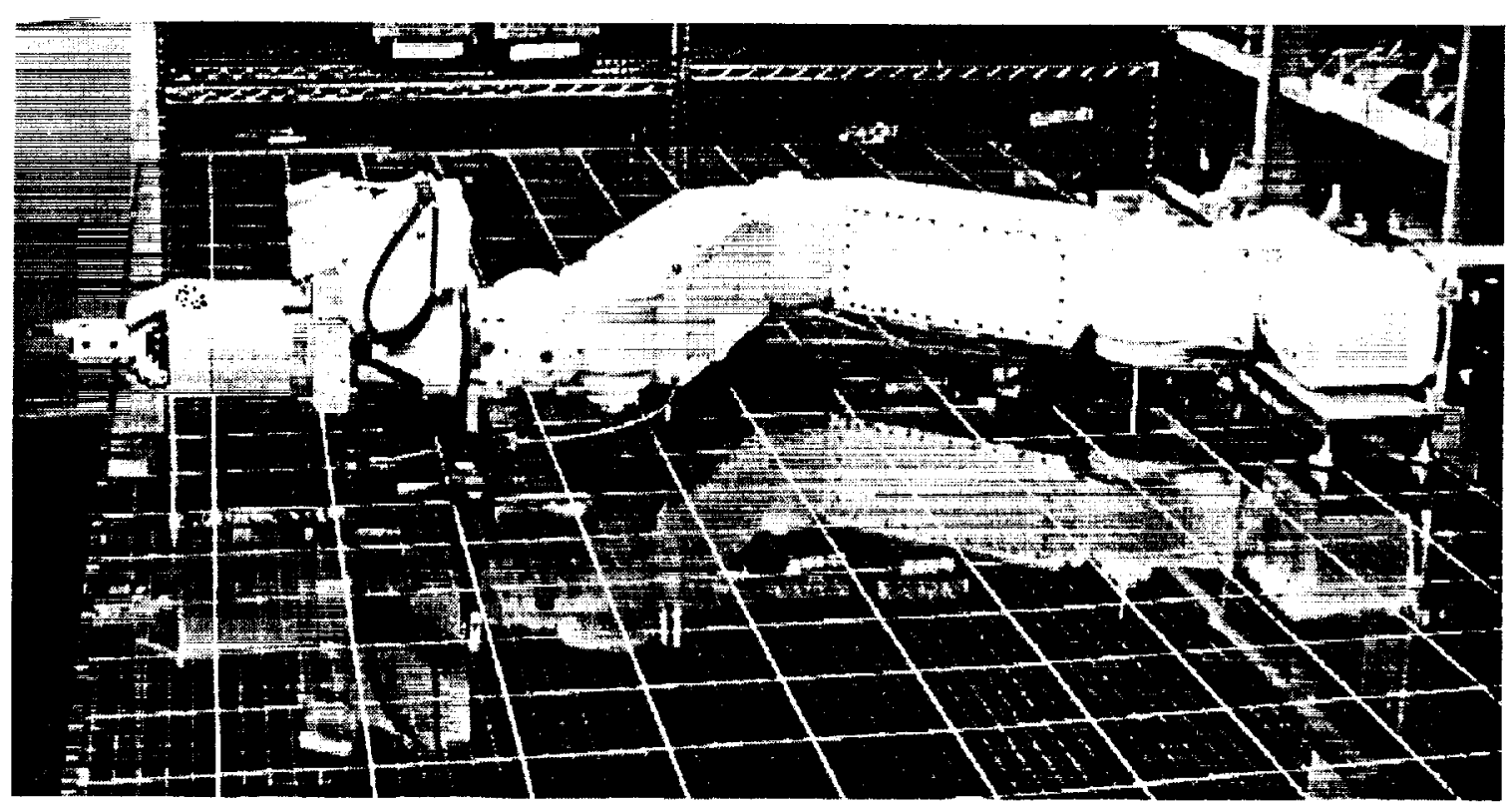

Figure 1 - FTS Manipulator on Air Bearing Table

Copyright 1093 by the American Instllute of Aeronautics and

Astronautles. Inc. No copyrlght is asserted in the Uniled Stales under Title 17, U.S. Code. The U.S. Government has a royalty-Iree license to exerclse all rights under the copyright claims herein for govermment purposes. All olher rights are reserved by the copyright owner. 
unlocking a SSF Truss Joint, mating/de-mating a fluid coupling, contact following of a contour board, demonstrating peg-in-hole assembly, and grasping and moving a mass. Future tasks foreseen for the FTS system included ORU (Orbit Replaceable Unit) change-out, Hubble Space Telescope Servicing, Gamma Ray Observatory refueling, and several insitu SSF servicing and maintenance tasks. Operation of the FTS was planned to evolve from teleoperation to fully autonomous execution of many tasks. The FTS manipulator has been assembled at Martin Marietta (see Flgure 1) and will be delivered to NASA/JSC (Johnson Space Center). Successful component tests indicate a manipulator which achieves unprecedented performance specifications.

Currently anticipated tasks for dexterous space manipulators still focus on reducing EVAs as well as enhancing crew activity and safety. The Space Station (Freedom ?) plans to utilize a dexterous manipulator, SPDM (Special Purpose Dexterous Manipulator), on the SSRMS (Space Station Remote Manipulator System) to perform maintenance tasks such as replacement of ORUs. A potential being Investigated for use on the Space Shuttle in assistance with EVA worksite setup and teardown. The first and last portion of most EVAs consist of placing or retrieving PFRs (Portable Foot Restraints), Tool Boards, and other devices needed to support EVA tasks. Other possible uses for dexterous manipulators include contingency use to avoid additional EVA crew intervention. The FTS manipulator requirements and designs are examples by which to assist in understanding current dexterous manipulator tasks and plans.

The wide range of FTS mission tasks combined with the desire to evolve toward full autonomy forced several extremely demanding requirements. Some of these requirements may be excessive to telerobotics community, but the FTS requirements appear to have been created to accommodate an open-ended evolution. This operational evolution would not be Impeded by functional limitations in the FTS manipulator systems. Many of the FTS requirements discussed in the following sections greatly influenced the development cost and schedule of the FTS manlpulator. A recommendation arising from the FTS program to remedy the possible Impacts from such ambitious requirements is to better analyze candidate robotic tasks. Based on these task analyses, then weigh the operational Impacts against development impacts prior to requirements definition.

\section{EunctlonaL Requilements}

The functional requirements of the FTS manipulator involve environmental, performance, safety, and resource effects. Many of these requirements are driven by the space environment, such as operation in thermal extremes, the need for safety, and limited resource avallability (weight and power). Many of these requirements, however, focus on the manipulator and component functions to insure superior performance and ability to upgrade (evolution toward autonomy).

The primary robotic function of the FTS manipulator is that it move or manipulate objects in zero-gravity. Because interchangeable endeffectors were being considered, the manipulator requirements specify the tool-plate as the point of reference (see Figure 2 for FTS manipulator dimensions and components.) The tool plate is the attachment point for the wrist force/torque sensor. A manipulated object's mass may be as high as 37 slugs (1200 lb.) with the manipulator able to move masses less that 2.8 slugs $(90 \mathrm{lb}$.) at velocities of $6 \mathrm{inch} / \mathrm{second}$. Unloaded tool plate velocity will be at least 24 inch/second. Accuracy of tool-plate positioning relative to the manipulator base frame must be within 1 inch and \pm 3 degrees. The manipulator must be able to resolve tool-plate incremental motion within 0.001 inch and 0.01 degrees. (Of coarse, verification tests of such extreme resolution specifications is costly.) Additionally, repeatability must be within 0.005 inch and \pm 0.05 degrees with respect to the manipulator base frame. To perform useful work, the FTS manipulator was required to provide 20 pounds force and 20 foot-pounds torque output at the tool plate in any direction and in any manipulator configuration. These output force and positioning requirements were to be utilized with several control schemes including joint-by-joint, Cartesian, and impedance control.

To operate in space, the FTS manipulator had to meet the shuttle safety requirements as well as the environmental extremes. The safety requlrements, as discussed later in this paper, ensure Orbiter and crew safety through fault tolerance. Safety is cited by Shattuck and Lowrie [1992] as "the single largest factor driving the system design." Safety and fault tolerance requirements resulted in monitoring of joint and Carteslan data, in checking of loop times to ensure proper functioning, in cross-strapping along communication paths, and in the addition of a hardwire control capability as a backup operational 
mode. Orbiter launch and landing Impart vibration into the system which requires structural analysis and testing. Electromagnetic interference (EMI) must be limited both from Invading and from exiting the manipulator systems. However, the most demanding aspect of the space environment from the FTS designer's view is the thermal vacuum of space. Operation in a hard vacuum $\left(10^{-5}\right.$ torr) and over temperatures from $-50^{\circ} \mathrm{C}$ to $95^{\circ} \mathrm{C}$, with directional heating and gradients, forced innovative designs, careful material selection, and extenslve analysis.

Another consequence of the space environment is operation in zero-gravity. Designing the manipulator for a zero-g environment impacts structural, electromechanical, and electrical power considerations and well as the control system design. Because weight is a premlum in space, motors are chosen to provide torque's for zero-g operation. This saves significant weight and electrical power when compared to motors chosen for ground-based operation. Smaller motors also benefit the themal control system. The structure must also be lightweight, which increases flexibility and lowers structural bending mode frequencies. While being lightweight and more flexible, space manipulators are expected to handle payloads more massive than the manipulator. This expectation is far different from terrestrial manipulators which usually handle payloads less than $1 / 10$ the manipulator weight. To maintain stability and performance of the FTS system, a 10:1 ratio is maintained between the first bending mode and the control bandwidth. This ratio precludes use of high bandwidth PID servos used In more massive, terrestrial manipulators. To address the stabllity and performance issues in the FTS manipulator, the structure was designed for stiffness $(12 \mathrm{~Hz}$ first bending mode) and the manipulator control has a $1.2 \mathrm{~Hz}$ bandwidth, an inertia decoupler, and joint-level position, velocity, and torque servo control loops.

\section{Manipulator Deslon Technologles}

Beyond safety, FTS manipulator design was driven by the thermal environment and the positioning performance specifications. Of course, each manipulator subsystem was influenced by additional constraints and specifications. The following paragraphs describe the manipulator subsystem designs and technologies developed by Martin Marietta and its subcontractors to meet the FTS requirements and specifications. Manipulator subsystems discussed include manipulator kinematic design, link structure, actuators, control systems, and the end-of-arm tooling.
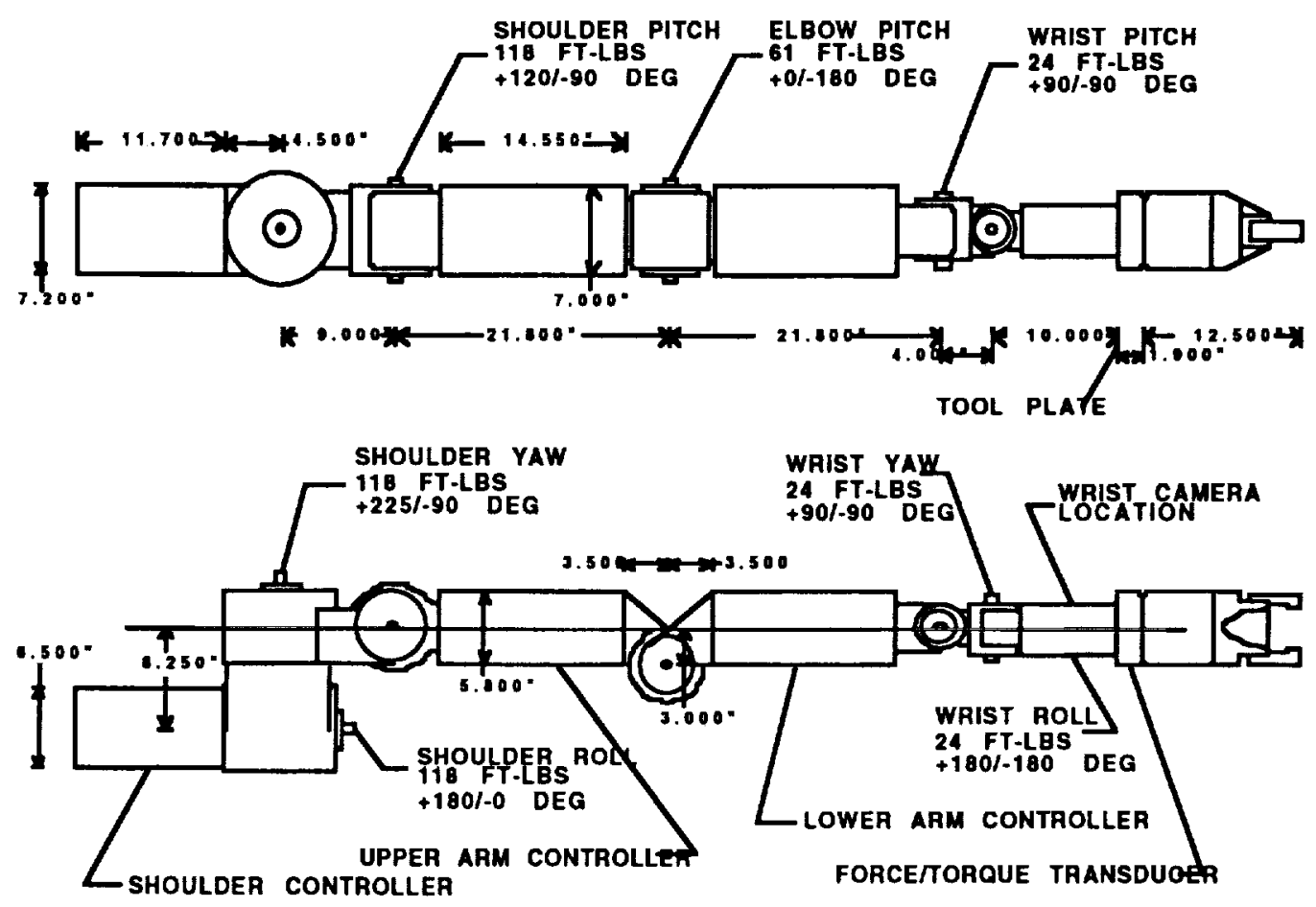

Figure 2 - Manipulator Dimensions \& Components 
Manipulator Kinematics

A 7-DOF (degree-of-freedom) R-Y-P-P.P-Y-R design is used with the first joint (shoulder roll) utilized for task-dependent conflguration optimization. The outer 6 joints are actively controlled for coordinated output motion. The kinematic design has minimal joint offsets and $90^{\circ}$ twist angles to simplify the kinematics. The 6-DOF kinematic arrangement, with three adjacent pitch joints, provides a closed-form inverse kinematic solution with fow singularities within the manipulator workspace. The singularities which occur when the wrist roll or wrist yaw align with the shoulder yaw are beyond the usual workspace of the manipulator. Other singularities occurring at joint limits and when the elbow passes over the "home" position (see Figure 3), shown below, are eliminated with mechanical and software joint travel limits. The 3 inch displacement of the elbow joint is to allow the arm to fold back on itself for a greater workspace.

\section{Link Structure}

The manipulator links provide structural support as well as joint controller electronics packaging and thermal control. Packaging and thermal control determined link sizes while fracture and stiffness considerations drove the structural design of the links. A stiffness requirement of $1,000,000$ pounds/foot and 1,000,000 foot-pounds/radian resulted in a smallest structural safoty margin which exceeds 14, far greater than Shuttle requirement for a 1.4 factor of safety. Easy access to electronics is through side plates on the links. To avoid the cost and complication of active cooling, radiation is the primary thermal path. The controller boards sit in slots within the links which provide conduction paths to the link structure for radiation to the environment. Figure 4 shows the links and the computer cards which fit within the links. The link designs use material coatings, mounting hardware, and Kapton/lnconel film heaters to maintain thermal control.

Actuators

The joint actuator designs, developed by Martin
Marietta and Schaeffer Magnetics, were also driven by positioning, performance, and thermal demands. These high-performance, zero backlash actuators each house a DC-motor, harmonic dive transmission, output torque sensor, output position sensor, fail-safe brake, hard-stops, and internally routed cabling. The design achieves considerable commonalty between actuators. Three sizes are used - one for the 3 shoulder joints, one elbow jolnt, and one for the 3 wrist joints.

The DC-motors have brushless, delta-wound stators with samarium cobalt rotors. This design offers good thermal properties, low EMI, minimal rotational losses, and linear torque-speed relationships. Motor commutation signals are generated from Hall Effect sensors, a second set of which is Installed for redundancy. A secondary set of windings within the stator, driven via an independent electrical path, provides at least $10 \%$ rated torque and 0.5 degrees/second joint velocity for operation of a backup mode. This degraded mode of operation, commanded joint-by-joint satisfies the need for safing the manipulator after failure of a primary system. Fail-safe brakes attached to the motor rotor shatt are spring-loaded so that loss of power engages the brake. These brakes may be released with an EVA release bolt, which when tumed $90^{\circ}$ releases a cam on the brake armature.

Harmonic drives provide 100:1 backdrivable gear reduction in a compact volume. The harmonic drives were chosen for torsional stiffness and zero backlash. Cup size is determined by joint torsional stiffness requirements. In fact, because of the relative flexibility of the harmonic drive, all other torsion members are considered rigld. Rather than the standard Oldham coupling to the wave generator, a specially designed cylindrical coupler was used to eliminate backlash. Additionally, the output is coupled to a flange around the motor and harmonic drive. This flange, mounted to large duplex bearings provides compactness, rigidity, and an efficient load path to the output link.

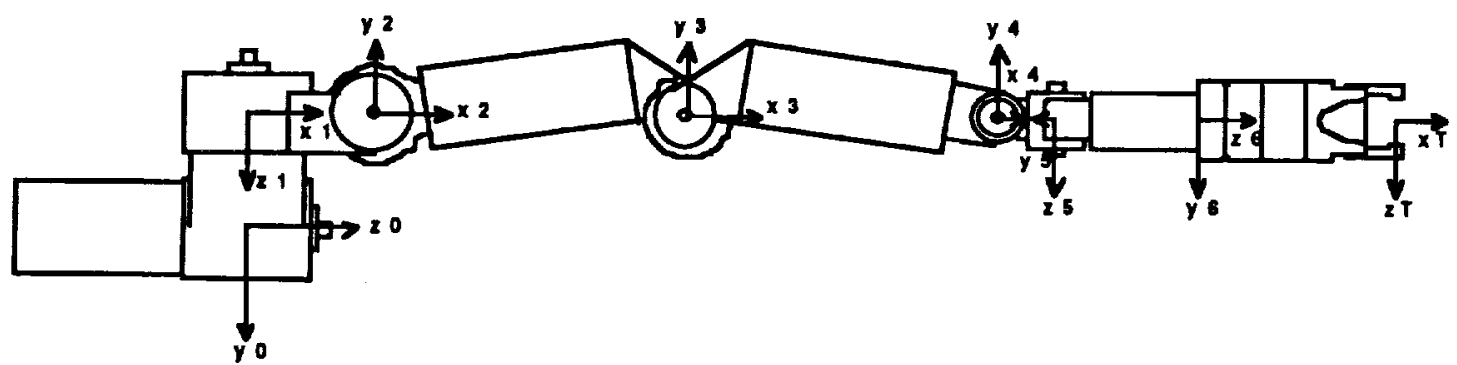

FIgure 3 - FTS Manlpulator "Home" Position 
An analog torque loop is implemented in the joint servos to accommodate the non-linear and highfrequency affects of the harmonic drives. Sensed torque values come from an output torque sensor embedded on the hamonic drive output flange. Strain gages are mounted to the spokes of the titanium flange. This sensor placement isolates the sensor from structural loads (bending), thus primarily transmitting actuator forque. For effective performance, this analog torque loop operates at $1500 \mathrm{~Hz}$.

Like the manipulator structure, the actuator housings and bearings were designed for stiffness and thermal stability. A standard bearing steel, $440 \mathrm{C}$ stainless, is used for all bearings. Bearing lubricant is Braycote 601, a liquid lubricant used in space applications. Its very low vapor pressure allows the actuator to be vented rather that sealed, but was still designed to resist contamination and assembly in a clean room. The motor bearings are deep-groove roller bearings sized for the thrust load of brake engagement and spring pre-loaded to minimize temperature sensitivity. The output bearings are large diameter, duplex-palr, angular contact bearings (face-to-face mounting). These bearings share radial and thrust loads with another duplex-pair on the other side of the actuator. An exception is the wrist roll, which has a single, duplex pair mounted back-to-back for better rigidity against the bending moments of the full cantilever load. Unfortunately, this back-to-back Installation has greater sensitivity to assembly misalignments. This sensitivity may contribute to the excessive, uncompensated friction discovered during recent wrist roll torque loop tests.

The actuator housings are aluminum and titanium. Titanium is utilized near bearings. The similar thermal properties of $440 \mathrm{C}$ stainless and $6 \mathrm{Al}-4 \mathrm{~V}$ titanium minimize temperature effects on bearing pre-loads. These pre-loads were determined as a compromise between stiffness and friction drag. The actuator case was designed for thermal needs. Motor and brake heat is dissipated to the ends or to the casing and then radiated to the environment. Like the links, the actuator design uses thermal isolation, material coatings, and internally mounted film heaters to protect bearings from thermal gradients. These gradients could adversely affect actuator friction and positioning accuracy.

The positioning and incremental motion requirements call for encoder data within an arcminute which required position resolutions to 22bits. To meet this need, inductive encoders were developed specifically for the FTS program by Aerospace Controls Corporation. These encoders have a fine and a coarse track used for Incremental and absolute position resolution, respectively. Temperature effects on sensor accuracy were discovered during thermal testing. These errors were stable and repeatable with temperature, and are thus correctable in software.

All cabling in the manipulator is Internally routed through the links and actuators. Each actuator has a cable passageway designed to eliminate twisting

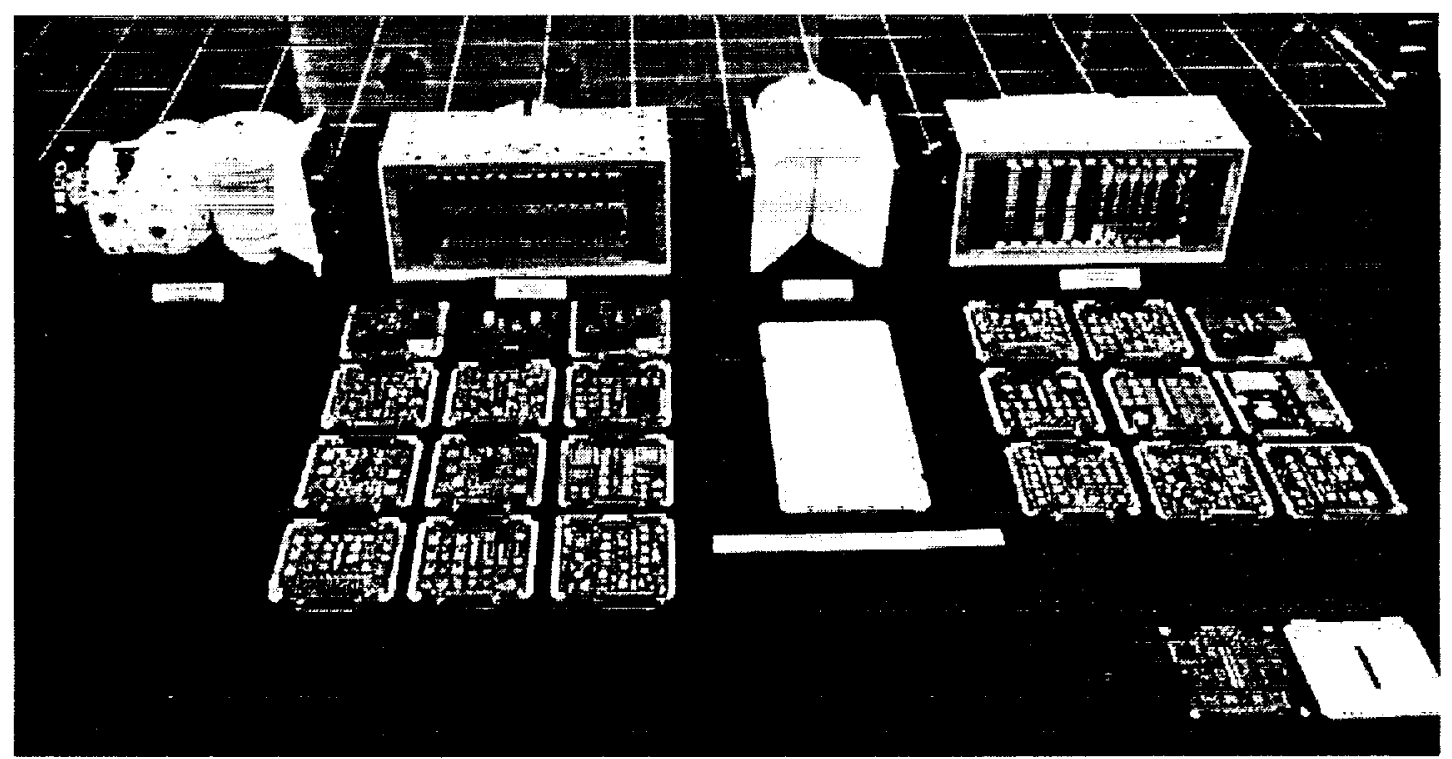

Figure 4 - FTS Manipulator LInks and Controller Cards 
of cabling, thus minimizing chafing opportunity. The Innovative cabling within these actuators is of Flat Conductor Cables (FCC), manufactured by Tayco, Inc. FCC is used in space applications, but for this application up to 34 layers of laminated cables are used in a single actuator passageway. The cables consist of altemating layers of Kapton, FEP, and photo etched copper conductors with a vapor-deposited copper shield. These cables are to operate from $-50^{\circ} \mathrm{C}$ to $95^{\circ} \mathrm{C}$ through thousands of cycles. These cables route serial data, video signals, power, and discrete signals. Acceptance tests of a few cables indicated minor lamination problems apparently due to entrapped water vapor. Investigation of the cable manufacture and tests of additional cables indicated several areas for possible change as well as a method for cable repalr. Recent cable tests to 100,000 mechanical cycles over full temperature ranges verified continued cable functionality.

\section{Control Systems}

The FTS manipulator control design provides 6DOF active control over a wide range of payloads as well as Impedance control for stable contact. The wide payload range specified for the FTS manipulator causes the manipulator joints to experience inertial loads over several orders of magnitude. These loads are induced by the coupling which occurs between joints and affects the trajectory-tracking accuracy of the manipulator. The position controller implemented in the FTS manipulator compensates for these torques with a model-based inertia decoupler. The feed-forward decoupling scheme computes expected inertial torques due to commanded motion and sums this torque with the jolnt command. The positiondependent inertia matrices used to calculate these torques are computed every $200 \mathrm{~ms}$, a time chosen as a compromise of accuracy and computational burden.

In addition to the free-space performance requirements, satisfied with the position controller and inertial decoupler, the FTS manipulator must provide stable contact with its impedance control (see Figure 5). The impedance controller is position-based, that is, the manipulator and joints are treated as actuators of Carteslan position. Thus, end-effector force measurements are transformed into Cartesian motion commands based on a desired output impedance. To maintain stability during the transition from freespace motion to contact, a joint velocity foedback term is included for "augmented damping." The resulted lightly damped contact insures stability, but when contact is broken the free-space motion becomes overdamped and sluggish. A feedforward velocity term is implemented to compensate for this poor free-space response. These control schemes, which increase the complexity of the controller are designed to meet the FTS free-space motion, payload capacity, and contact performance requirements.

\section{Emergency Shutdown}

An emergency shutdown (ESD) system is embedded in the manipulator control architecture. This system was implemented to provide active control of hazards to meet the payload safety requlrement to be two-fault tolerant against catastrophic hazards. The primary hazards in this case are unplanned contact and excessive force generation. The ESD approach is to use 3 control levels to monitor joint and Cartesian positions and velocities, comparing both commands and sensor feedback. A separate ESD bus, which connects the joint, manipulator, and power controllers, is the path by which an ESD is initiated - removing power from the manipulator systems. The first level checks that commanded values are within allowable limits both in the manipulator controller and the joint controllers. The second level monitors safety critical parameters such as position, velocity, and torque with the joint controllers and within the manipulator controller collision avoldance routines. The final level of ESD monitoring is a check of redundant safety critical

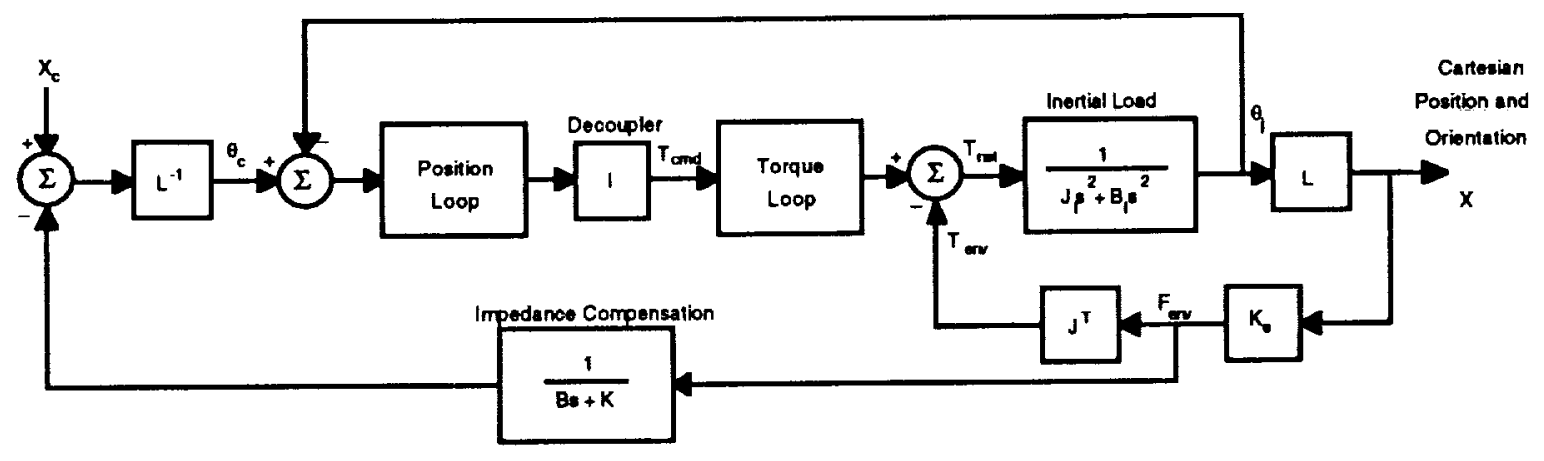

Figure 5 - Manlpulator Impedance Control Block Diagram 
parameters in the redundant manipulator controller and in independent joint controllers.

In the event of an apparent failure, several possible ESD actions may be automatically initiated. The operator, of course, has a manual ESD to power off the manipulator at any time. If monitored values are elevated but do not pose immediate danger, a soft stop is initiated by the control software. A soft stop commands the manipulator to hold the current position with brakes off (disengaged). An example of a soft stop condition is a Cartesian manipulator command which violates a warning boundary near a known obstacle. A hardware ESD is initiated by any controller when an analog sensor value exceeds its limit value - resulting in an ESD notification on the ESD bus. These analog comparisons are being performed at $1500 \mathrm{~Hz}$. A software ESD occurs when a controller CPU detects an out-of-limit condition and signals the

power module over the Mil-Std-1553B communication bus. The power module then Initiates a combination ESD to power off the manipulator. A combination ESD is detected by software comparisons in the controllers and initiates a software reset of a hardware limit value to force a hardware ESD. All these ESD paths were analyzed to determine reaction times to various failures such as a joint runaway. Hardware ESDs occur in $11 \mathrm{msec}$, combination ESDs occur in 30 to $206 \mathrm{msec}$, and a combination ESD may take up to
4026 msec for an over-temperature condition.

\section{Gripper/End-of-Arm Toolino}

The end-of-arm tooling built for the FTS manipulator has a parallel jaw gripper and space for later addition of an end-effector exchange mechanism. This gripper and wrist mounted camera and lights are shown in Figure 6 . The gripper fingers are a cruciform designed for positive contact and retention. The gripper fingers ride on a rack and pinion driven by a harmonic drive transmission and a single DC-motor. A pair of failsafe brakes are installed to provide fault tolerance against inadvertent release. Each of the two brakes can withstand forces greater than expected gripper forces (maximum anticipated load is $30 \mathrm{lb}$, brake hold is $50 \mathrm{lb}$ ). Gripper forces are measured by a torque sensor and also by motor currents. The concern over inadvertent release also impacted the design of planned task items. These items were instrumented to insure positive grasp. As a final safety measure, the gripper fingers are attached with EVA compatible bolts which may be removed on-orbit to release the gripper.

\section{Safety Requirements}

Robotic Manipulator Systems can provide the capability to perform work and assist humans in space as long as they are safe and reliable. The space based requirements differ significantly from

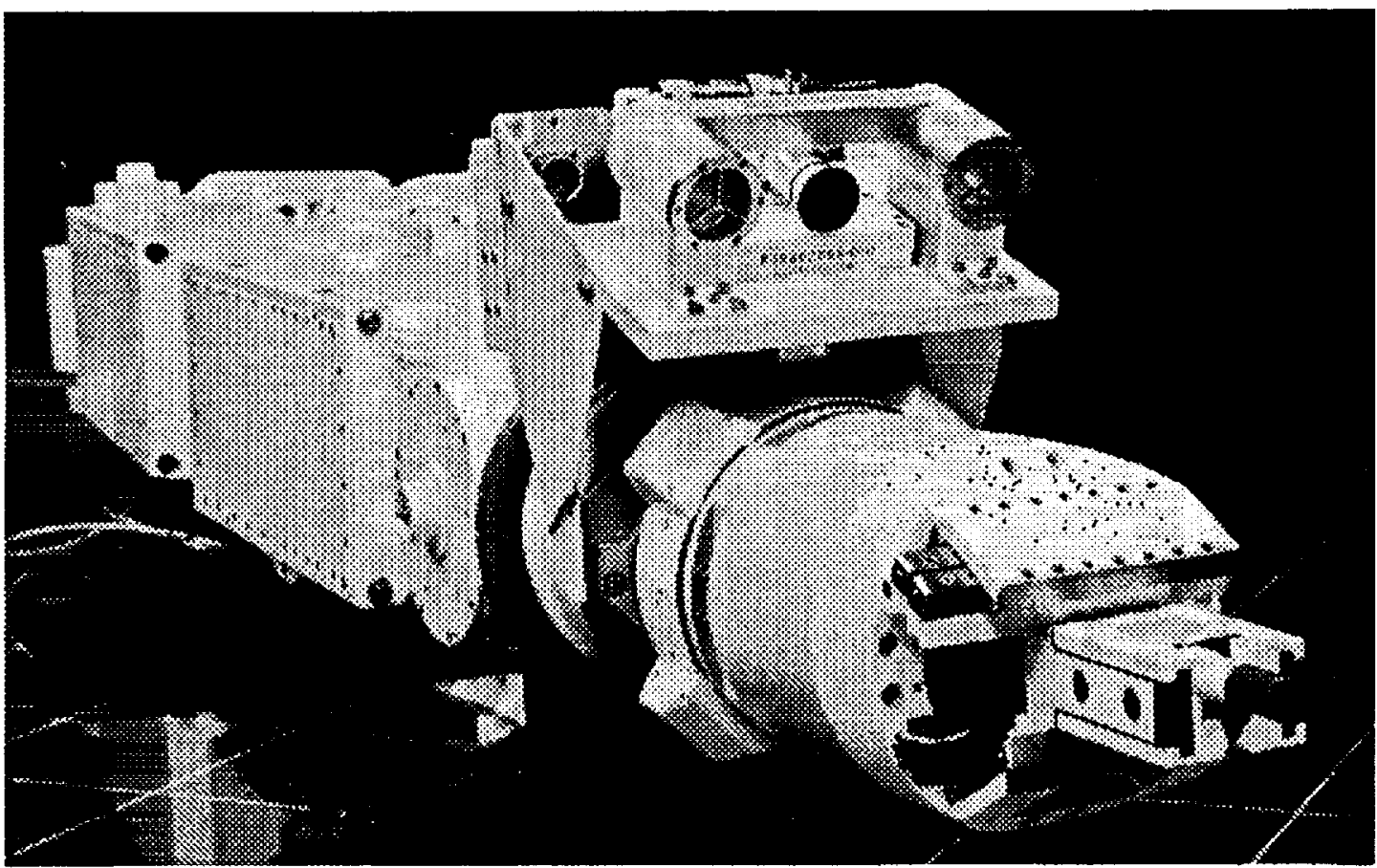

Figure 6 - End-of-Arm Tooling/Gripper 
terrestrial based manipulators used in industry and research. In most terrestrial robot implementations, the prime method for dealing with failures is to keep workers out of the robot workspace when active and by accepting the occasional parts damage following a failure due to high volume parts fabrication. This approach is not acceptable for space applications where humans are involved, the effects are very high in costs or It's extreme difficulty to repair. These effects impact the design requirements for space manipulator systems.

\section{Hazards and Controls}

All manned space flight systems are assessed for flight hazards their use would impose. From such an assessment the causes of those hazards are determined, and methods to control those hazards are developed. To gain flight acceptance, multiple levels of hazard control must be designed and verified to assure the desired level and coverage of controls. In the FTS system development, safe control of hazardous operations forced additional requirements in the design of the manipulator system, its intertaces with the Orbiter and the task elements the FTS was to interact with.

The primary hazards associated with the FTS manipulator operations and the three methods for providing safe control are as listed:

A) Unplanned contact or impact during operations

1) Operator and computer control to not command unplanned contact.

2) Boundary management software operation.

3) Redundant boundary management software operation in the safety computer

B) Inadvertent release of hardware

1) Hardwired enable gripper brake power from Independent switch in the aft flight deck

2) Operator Interface Computer: (the aft flight deck portable laptop computer) command to release gripper Brake \#1

3) Hand controller switch to release gripper Brake \#2

C) Failure to stow for safe Orbiter landing

1) Nomal computer operations (With hardwired control for added rellability)

2) Jettison via RMS (or EVA if time permits)

3) EVA operations to stow or jettison

D) Excessive applied gripper force or torque

1) Force control using gripper force sensor

2) Current limiting ESD (Emergency shutdown detection)

3) Redundant current limiting ESD

E) Excessive applied manipulator force or torque

1) Normal control with active Carteslan load from joint torque command
2) Cartesian force limiting, using wrist force/torque sensor channel A

3) Redundant Cartesian force limiting, using wrist force/torque sensor channel B.

\section{Mission Operation To Control Hazards}

Primary concerns in the design of space manipulator systems have to do with the effects of system failures on the crew or vehicle. Operational limitations of use are placed on robotic systems that may otherwise be perfectly capable of performing their Intended operations. Limitations on use are imposed due to the fact that if a system is pertorming a task and were to have a failure, the effect of that failure must not prohibit the intended function from being performed in the time frame that function is critically needed, and any fallure must not prohibit any other safety related operations from being carried out during its time of criticality.

For a system to continue operations after a failure, any remaining operability the system might contain must also provide that same capability to make itself safe to the vehicle and crew if it were to suffer a failure. Otherwise that additional level of operability would only be allowed for temporary use to make the task situation safe, remove the robot from the task area, and then stow it in a safe retumable state or eject it so the vehicle can retum to Earth. The added operability would not be allowed for continued use to proceed with the intended task, except to make the situation safe. This is the fundamental concept of hazard control for the Orbiter.

\section{FTS Fail Safe Operations}

Several FTS configuration descriptions follow below along with design features to address key functions which allow for safe operations. The designs comply with NASA's Orbiter safety policy and requirements of NSTS $1700.7 \mathrm{~B}$ with interpreted in NSTS 18798A. In several cases, the hardware or software system could not be designed to meet the required levels of fault tolerance without significantly complicating the design or dexterity of the manipulator system. Therefore reductions in compliance with the safety requirements placed operational limitations on the use of the FTS System. The system is considered fail safe; where under any failure the system will not cause a catastrophic hazard, and therefore does not jeopardize the safety of the Orbiter or crew. The FTS system is not fail-operational. Such a sysfem, after any initial failure, could continue normal intended operations since it would still retain the ability to make itself safe after a second tailure. 
The DTF-1 concept fulfills the first method of hazard control for Obiter safety using its normal modes of operation. If any of the single points of failure occur, normal operations will cease and an attempt to safe the manipulator system by use of the hardwired control. Note that hardwired control is only a supplement to the first level of hazard control. If the manipulator system cannot be safed by use of the hardwire control, the mission will be assessed to determine if enough time remains to perform an EVA to safe the manipulator system. If hardwired control cannot safe the manipulator system and time does not permit an EVA to safe the manipulator or remove it for stowage, then the RMS will grapple the telerobot using the RMS grapple fixture for jettison. This is the second method for hazard control. The third method of hazard control to provide two fault tolerance for Orbiter safety is EVA operations. Remedial operations could be to remove the manipulator, release the gripper and/or release the actuator brakes. This would be to allow stowage of the manipulator, either into its caging devices or by removal and strapplng it in the airlock, or otherwise by release into orbit.

\section{Hardwired Control}

The FTS system incorporates a backup hardwired control capability in the event of a failure which precludes closed loop computer control of the manlpulator system. The main purpose is to minimize the likelihood of having to jettison the system or perform an EVA operation. This has the effect of making the computer system, sensor systems, software, servo systems and most other hardware single fault tolerant, even though the operations would be significantly degraded in performance.

Operational use of the hardwired control is limited to safing of the system after a failure, by stowing the arm to allow a sale Orbiter retum. It allows operator control of individual manipulator joints for stowage and for gripper actuation in the event of computer control or motor drive failure. When selected, primary power is removed from all manipulator motor and brake drivers while retaining power to camera controls. Software recognizes the status of the hardwire control, and commands off all motors and brakes, so that retum to normal computer operations after hardwired control starts with all motors and brakes powered off.

Hardwire control is limited to very low joint rates and torques. Hardwired control is by sequential, Jointby-jolnt movement, and provides no force accommodation to minimize forces imparted into interfaces. Only a limited set of initiated tasks are likely to be able to be completed. Emergency shutdown detection (ESD) is not operational during hardwired control operation, as the operator can de-power the hardwired drive to stop payload motion, and brakes can also be used to stop motion.

\section{EVA Operations}

Several failures of components employ EVA as the third hazard control path to ensure stowage of DTF-1 for safe return of the Orbiter. The manipulator actuators, gripper mechanlsm, and manipulator caging mechanisms represent major groups of such components.

Failure of a caging mechanism to release the arm for operation would not require EVA for safing the manipulator. EVA would be used as the third path for safing the manipulator if more than one of the four caging mechanism fail to close. In this case, removal of the manipulator at its shoulder interface and either manual release into orbit or stowage in the aillock would be required.

Failure of a manipulator actuator motor drive electrically or mechanically would require EVA as the third controlled path. Mechanical release of the joint actuator brake allows EVA backdrive of the joint into the caging position. If a manipulator joint seizes, then EVA is employed as the third hazard control path to remove the manipulator at the shoulder and release Into orbit or stowage in the airlock.

\section{Single-Points Failures:}

There are several single point failures that remain in the FTS system which may lead to failure of the manipulator to complete a task, or to stow thself for a safe Orbiter return. For the Orbiter this is considered a catastrophic hazard, therefore the requirements for payloads to provide two fault tolerant methods of dealing with these effects.

The FTS single-point failures which lead to an EVA or jettison are few in function, but have commonalty within the actuator and gripper. These failures are seized bearings or gears, a short within the motor winding, or a short or open in a brake winding.

\section{Safety Critical Subsystems}

The DTF-1 Flight Experiment of FTS has fifteen different safety critical subsystems and equipment groups, as listed:

Structure Subsystem, Manipulator, Controls, Data Management and Processing, Vision, Sensors, Software, End-of-Arm Tooling, Electrical, Power, 
Electromechanical Devices, Thermal Control, Task Panel Elements, Aft Deck Workstation, and Hand Controllers

\section{Current Status}

The flight FTS manipulator assembly and initial tests were completed under the FTS Technology Capture program at Martin Marietta Astronautics, Denver, in July 1993. An acceptance test and demonstration occurred July 28, 1993, with NASA participation by JSC and LaRC. The tests were conducted on an air-bearing table with all seven joints active, but only four commanded to move for joint and coordinated Cartesian control. The joint servo controller loops had not been individual tuned, and therefore this testing constituted only a demonstration of operation, rather than a performance test. Contact stability and variable compliance interactions with external structures were also demonstrated. The servo tuning can be readily accomodated, as all parameters are programmable, including the torque loop frequency responses.

A follow-on effort called the Bridge Task integrated and checked-out the flight End-of-Arm Tooling (EOAT or gripper) and wrist camera onto the flight manipulator. The Mil-Std-1553B communications bus underwent performance tests between the three internal arm control computers and external coordinating controllers. Martin Marietta provided engineering assessments for a proposed flight experiment concept that separated the manipulator arm from the main avionics. The integrated safety design and control of the system was meticulously maintained. All engineering, analysis, data files and article data packages are being completed and documented under the guidance of Martin Marietta's QA and NASA's SR\&QA to maintain the flight heritage of the manipulator and components.

\section{NASA Fllaht Plans}

JSC developed an Oচiter based flight experiment concept to demonstrate a dexterous robotic manipulator system that can operate on the end of the Shuttle Remote Manipulator System (SRMS). This configuration was recommended by Shuttle payload and operations managers as the most useful and beneficial, as opposed to a relocateable dexterous device only. The operational uses allow planned payload manipulation tasks and provides a capability for contingency operations for payloads and for some Orbiter problems. The benefit is to minimize overall EVA time currently consumed by routine tasks, such as EVA site setup and takedown. This would allow EVA to be most usefully allocated for complex operations. Langley Research Center and JPL are team participants in this proposed venture, called DOSS for Dexterous Orbiter Servicing System. Langley would be responsible for advanced robotic controls development and JPL for advanced operator control from a ground control station.

The other significant function of DOSS includes ground control of the dexterous manipulator using 3-D graphic simulations in predictive displays to compensate for the time delays. Ground control allows multiple rotations of ground controllers to operate the dexterous manipulator. The flight experiment concept is cost effective, in that the most expensive development item, the flight manipulator, is available and can be capitalized on. The manipulator along with all ancillary avionics and mechanisms were designed to meet the integrated and operational Orbiter payload safety requirements. Such a flight experiment would provide significant risk mitigation for robotic applications in space, e.g. the new space station, since much of its maintenance is now baselined with the use of ground controlled robotics. The station program seems to be counting on dexterous robotics with no flight operations time to provide insight into possible complications.

\section{REFERENCES}

Shattuck, P. L., and Lowrie, J. W., Flight Telerobotic Servicer Legacy, SPIE Vol. 1829 Cooperative Intelligent Robotics in Space III, 1992, pg. $60-74$.

Andary, J. F., Hewitt, D. R., and Hinkal, S. W., The Flight Telerobotic Servicer Tinman Concept: System Design Drivers and Task Analysis, Proceedings of the NASA Conference on Space Telerobotics, Vol. III, January 31, 1989, pg. $447-471$. 
Flight Telerobotic Servicer Development Test Flight (DTF-1) Final Report, MD-15, Martin Marietta Astronautics Group, submitted to NASA Goddard Space Flight Center under contract NAS5-30689, 1991.

Space Station Flight Telerobotic Servicer (FTS) Phase C/D DTF-1 Phase II Safety Compliance Data Package for Payload Design and Flight Operations, 01-PA-32-08, Martin Marietta Astronautics Group. submitted to NASA Goddard Space Flight Center under contract NAS5-30689, August 1, 1991.

Space Station Flight Telerobotic Servicer (FTS) DTF-1 System Critical Design Review, 01-MD-02-CDR-02, Martin Marietta Astronautics Group, submitted to NASA Goddard Space Flight Center under contract NAS5-30689, October 2, 1990.

Space Station Flight Telerobotic Servicer (FTS) Design Criteria Document, 87600000005 Rev. N, Martin Marietta Astronautics Group, submitted to NASA Goddard Space Flight Center under contract NAS530689, March 23, 1990.

Space Station Flight Telerobotic Servicer (FTS) DTF-1 Manipulator Subsystem Specification - Final, 01TR-20-MS-06, Martin Marietta Astronautics Group, submitted to NASA Goddard Space Flight Center under contract NAS5-30689, December 4, 1991.

Space Station Flight Telerobotic Servicer (FTS) DTF-1 Control Stability Analysis and Simulation Report, 01-TR-05-02, Martin Marietta Astronautics Group, submitted to NASA Goddard Space Flight Center under contract NAS5-30689, October 11, 1990.

Space Station Flight Telerobotic Servicer (FTS) DTF-1 Control Stability Analysis and Simulation Report Preliminary, 01-TR-05-01. Martin Marietta Astronautics Group, submitted to NASA Goddard Space Flight Center under contract NAS5-30689, July 10, 1989. 